\title{
ON THE COMPATIBILITY OF THE APPROXIMATE PERRON AND THE CESARO-PERRON INTEGRALS
}

\author{
H. W. ELLIS
}

Saks calls two definitions of integration compatible if every function that is integrable in both senses is integrable to the same value in both senses. The purpose of this note is to give an example showing that the definitions of J. C. Burkill's approximate Perron (AP) [1] ${ }^{1}$ and Cesàro-Perron (CP) integrals [2] are not compatible. More generally the same example can be used to show that the definitions of integration that have been given for which the continuity of the indefinite integral is replaced by some kind of mean continuity are not compatible with those with continuity replaced by approximate continuity.

Definition. The function $F(x)$ is Cesàro continuous at the point $x=x_{0}$ if $F(x)$ is integrable in the Denjoy-Perron sense in the neighborhood of $x_{0}$ and if

$$
\lim _{h \rightarrow 0} \frac{1}{h} \int_{x_{0}}^{x_{0}+h} F(t) d t=F\left(x_{0}\right) .
$$

Burkill uses major and minor functions in defining the AP- and $\mathrm{CP}$-integrals as generalizations of the Perron integral. For a function $F(x)$ that has a finite derivative at all points of an interval $[a, b]$ except a finite or denumerable set to be an indefinite AP-integral (CP-integral) of any function coinciding with $D F(x)$, where $D F(x)$ exists, it is sufficient that $F(x)$ be approximately continuous (Cesàro continuous) on $[a, b]$.

For $n=1,2, \cdots, F_{n}(x)$ is defined to be zero everywhere except at the points of an interval $I_{n}^{\prime}$ of length $1 / 2^{n+1}$ strictly contained in $I_{n}=[1 /(n+1), 1 / n]$. On $I_{n}^{\prime}, F_{n}(x)$ is defined in such a way as to be non-negative, absolutely continuous on $I_{n}$, with a finite derivative on $I_{n}$ and with

$$
\int_{1 /(n+1)}^{1 / n} F_{n}(t) d t=\frac{1}{n(n+1)} .
$$

The functions $F(x), G(x)$, and $f(x)$ are then defined as follows: $F(x)=G(x)=\sum_{1}^{\infty} F_{n}(x), x>0 ; F(x)=0, G(x)=1, x \leqq 0 ; f(x)=D F(x)$ $=D G(x), x \neq 0 ; f(0)=0$.

Received by the editors June 8, 1950.

${ }^{1}$ Numbers in brackets refer to the references at the end of the paper. 
A simple computation shows that the density of the points at which $F(x) \neq 0$ is zero at $x=0$ so that $F(x)$ is approximately continuous at the origin and, since it is continuous by definition elsewhere, $F(x)$ is approximately continuous everywhere. The function $f(x)$ is therefore AP-integrable on $[a, b]$, where $[a, b]$ is any interval containing the origin, to $F(b)-F(a)$.

The function $G(x)$ is Lebesgue integrable on $[a, b]$ and for any point $x, 1 /(n+1) \leqq x \leqq 1 / n$,

$$
\begin{aligned}
\frac{n}{n+1} & =n \int_{0}^{1 /(n+1)} G(t) d t \leqq \frac{1}{x} \int_{0}^{x} G(t) d t \\
& \leqq(n+1) \int_{0}^{1 / n} G(t) d t=\frac{n+1}{n} .
\end{aligned}
$$

If we let $n \rightarrow \infty$, it follows that

$$
\lim _{x \rightarrow 0} \frac{1}{x} \int_{0}^{x} G(t) d t=1
$$

and therefore $G(x)$ is Cesàro continuous on the right at the origin. The function $G(x)-1$ serves as both a major and minor function to $f(x)$ on $[a, b]$ in the sense of Burkill's CP-integral, so that $f(x)$ is CP-integrable on $[a, b]$ to $G(b)-G(a)$, which differs by unity from $F(b)-F(a)$.

\section{REFERENCES}

1. J. C. Burkill, The approximately continuous Perron integral, Math. Zeit. vol. 34 (1931) pp. 270-278.

2. - The Cesàro-Perron integral, Proc. London Math. Soc. (2) vol. 34 (1932) pp. 314-322.

QUEEN'S UNIVERSITY 